\title{
Level of Mental Health Service Integration in Primary Health Care Units in Debre Markos Town, Ethiopia, 2014
}

\author{
Celia Sanchez ${ }^{1}$, Nurilign Abebe Moges ${ }^{2, *}$ \\ ${ }^{1}$ Public health department, Touro University, California, USA \\ ${ }^{2}$ Public health department, Medicine and Health Sciences College, Debre Markos University, Debre Markos, Ethiopia
}

Email address:

SANCHEZ@tu.edu (C. Sanchez), nure113@gmail.com (N. A. Moges)

\section{To cite this article:}

Celia Sanchez, Nurilign Abebe Moges. Level of Mental Health Service Integration in Primary Health Care Units in Debre Markos Town, Ethiopia, 2014. Science Journal of Public Health. Vol. 3, No. 2, 2015, pp. 181-190. doi: 10.11648/j.sjph.20150302.15

\begin{abstract}
Introduction: Mental health disorders in low and middle income countries contribute to roughly $10 \%$ of the global burden of disease. In rural areas especially, lower access to care and lack of health services contributes to failure to get proper care and treatment for conditions such as schizophrenia, depression, anxiety, and even suicidal ideation. In settings where resources are low, mental health services from primary health care settings would allow for better detection and intervention strategies. Thus, this study aims to assess the current level of mental health integration in the primary care setting as a baseline for future improvement. Methods: A cross sectional study design using a literature review was conducted on the current mental health status of Ethiopia as well as previous strategies for integration of mental health in primary care. Next, quantitative data was collected from 2 primary care units in Debre Markos town in order to determine mental health utilization under the current health strategies. Program information was collected from a health professional within each primary health care unit along with observational data and document review. Referral data from the Debre Markos reference hospital was also collected in order to assess referral utilization. Results: Attempts for mental health integration into the primary care system are evident through policies of the Federal Democratic Republic of Ethiopia. However, the implementation is recent and the capacity for training and services is low. Low levels of mental health integration were recorded in the primary care setting. Areas of improvement include education and training for primary health care worker and communication between primary and secondary health care systems. Conclusion and Recommendations: Training for mental health services in primary care units is poor. It is recommended greater education and training for the various disorders including epilepsy, substance abuse, and depression are addressed. In addition, funding is necessary to increase utilization, education, and to reduce stigma among mental health patients.
\end{abstract}

Keywords: Integration, Primary Health Unit, Debre Markos, Principles, Mental Health

\section{Introduction}

Mental health illnesses often exist as co-morbidities with other diseases such as HIV, podoconiosis, and tuberculosis [1]. In addition, underdeveloped countries and low income countries have a higher risk for prevalence of a mental health condition [2]. For example, in 2011 approximately 350-400 thousand Ethiopians were estimated to be living with epilepsy alone[3]. The diagnosis and treatment of mental health conditions such as epilepsy is under represented in these parts of the world due to lack of priority, lack of resources, or clinical training and background to treat mental health disorders. Integration of mental health in primary care system could lead to better recognition and treatment of disease, reducing the daily adjusted life years (DALYs) lost to these conditions [2].

In Ethiopia, there is only 1 psychiatrist per 6 million people, or about 36 in the country as of 2012 [4]. Prevalence for mental health disorders is estimated to be $15 \%$ for adults and $11 \%$ for children [4]. Training is available for health extension workers, however, no mental health training is available for primary care workers [5]. About 215 Health Extension Workers (HEWs) were trained in mental health in 2004 as part of a 16 package service set to expand primary care throughout the country [5].

There are limited studies on the integration of mental health throughout Ethiopia. Most of the studies occurred in a greater number of urban regions than rural regions such as the Jimma zone, Addis Ababa, and Gondar. In addition, there 
are very few studies on the utilization of primary care services in rural areas where cases of mental health disorders may be high.

One attempt to understand need was taken through a study of the Jimma Zone in Ethiopia. It was concluded that mental health integration into the primary care system in the Jimma zone would be beneficial to targeting greater populations. A study in Debre Markos was used to determine prevalence of services in the Debre Markos town. The proposed research project evaluated baseline mental health services in the city of Debre Markos to bring awareness to the potential gaps in coverage. The primary health care system may be the easiest way to identify patients with psychosocial needs as it is the first line of help for the major population [6]. The research identified the level of current mental health integration in the system and also determined if there is greater need for services in primary health care.

Furthermore, knowledge on the proposed study would bring awareness to the problem. However, this knowledge would have to be followed by training, education, and funding, as well as support from the local and federal governments to bring change. The study signifies a measurement of current health conditions and identifies areas for improvement in the health service sector.

\section{Literature Review}

Untreated mental disorders contribute to suffering, disability and economic loss and affect millions of people worldwide [6]. While prevalence is immense, few people receive the basic services they need to treat mental disorders [6]. Barriers to closing the gap in care include lack of capacity, "over-reliance on the medical model," stigma, and lack of widely accessible venues [7]. Further, mental health disorders and other diseases influence each other; failure to get treatment for schizophrenia or severe depression leads to failure for treatment for other co-morbidities such as cardiovascular disease or HIV [8]. Schizophrenics and those suffering from major depression have a 40-60\% greater chance of dying early from an untreated co-morbidity than the general population [8]. Many suffer from lack of productivity, social stigma, and furthermore, neglect to get treatment. In addition, suicide is the second leading cause of death in young people [8].

Globally, several low income and middle income countries have served as models for successful integration of mental health services into primary care. According to World Health Organization (WHO) and World Organization of Family Doctors (Wonca), integration of mental services into the primary care system is feasible and could allow for greater treatment for those in need [6]. For example, in Argentina primary care physicians (PCPs) provide the first steps in treatment, diagnosis, and rehabilitation for psychiatric disorders [6]. The PCPs have mental health specialists available for supervision and help with complex cases and a community center has been established for outpatient services [6]. The integration of services in the community and primary level of care the ability of patients to reside in the general population has led to greater demand for mental health services [6]. Approximately $56 \%$ of primary care workers can prescribe without restrictions, $40 \%$ with some for most countries. Approximately $26 \%$ of countries let nurses prescribe with restrictions (3\% without restrictions) [9]. The increase in primary care responsibilities for treatment indicates a shift in mental health care.

In Sydney, Australia PCPs provide mental health care for elderly patients [6]. The key to successful integration in this case is the coordination between mental health specialists, nurses, psychologists and geriatric psychologists [6]. An alternative method in Belize includes a nationwide initiative for psychiatric nurses to provide training to primary care workers [6]. The emphasis on community level care and mental health promotion has reduced psychiatric services, increased outpatient care, and fostered greater support for preventative care [6].

According to WHO and Wonca a process of skill building for primary care health workers over time would be best suited to countries with limited resources and limited availability of specialty care physicians [6]. Ten principles established by WHO and Wonca for successful integration into primary care include [6]:

1. Mental health for primary care must be included in policies and planning

2. Support for attitude and behavior change in addressing mental health issues is essential to protection of human rights.

3. Primary care workers need appropriate training.

4. Training for primary care workers must be feasible and limited.

5. Primary care workers must have access to mental health professionals for referrals and support.

6. Psychotropic medicines must be accessible and available through distribution from primary care facilities.

7. Integration is a continual process which requires developments, training, agreement and acceptance.

8. A mental health service coordinator is essential to driving integration forward.

9. Mental health integration requires the involvement of various stakeholders including community health workers, nongovernmental organizations, and volunteers.

10. A sustainable system requires financial and human resources.

Since 2003, WHO has assisted in training over 150 psychiatric nurses to deploy in Ethiopia and Nigeria [4]. The goal is to integrate mental health into the general care system. Data on current integration and effectiveness is minimal. Further research, such as in the town of Debre Markos, would help determine if the plan was implemented as proposed, and what gaps there may be in delivering mental health services. In the United States (US) intellectual and developmental disabilities (IDDs) and psychiatric disorders interfere with ability to work, be productive, and social and 
family life [10]. About $30-40 \%$ of people with IDDs also suffer from psychiatric disorders [10]. This figure may be higher in the underdeveloped world. The particular diagnosis of a patient leads to various services to becoming available to that patient, meanwhile others may not get the same options [10]. An integrated care system in this case would allow more services to be available based on signs and symptoms rather than a particular diagnosis leading to more people receiving the treatment they need [10].

In a study of refugees who had experienced traumatic life events, primary care was the easiest path to interception for mental distress [11]. However, the study also concluded the doctor or caregiver needed to initiate the conversation, and they needed to devote time to inquire about the patient's condition [11]. Factors such as the doctor looking too busy did not provide intimate space for conversation [11].

Currently, medical doctors in Ethiopia receive approximately $2 \%$ of their training mental health, nurses receive about $3 \%$ of training in mental health, and about 215 of the health extension worker training staff has received mental health training by the mental health hospital in Addis Ababa [5]. Other primary medical staff does not receive mental health training [5]

Primary health care clinics throughout Ethiopia consist of both physician based and non-physician based care centers [5]. Medications for mood disorders are available in only 50$80 \%$ of the physician based facilities [5]. Throughout Ethiopia, primary care physicians (PCPs) are allowed to prescribe drugs, while nurses are restricted. Drugs are available about one half of primary care clinics with a PCP [5]. Lack of treatment is influenced by poverty and stigma leading to failure to seek help [12].

Several factors affect one's ability to seek and adhere to treatment. A study of outpatients of Amanuel Psychiatric Hospital in Addis Ababa Ethiopia found overall 51.6\% of the patients demonstrated a low resistance to stigma and concluded high stigma could ultimately affect the standards of care between patients [13]. While this study targeted patients already seeking help, research has linked lack of employee exposure to greater stigma in other health scenarios and thus lower quality of care $[14,15]$. A study in the Jimma region found $7.4 \%$ of patients on antiretroviral medication dropped out of treatment due to mental illness [16]. In a study of 845 participants within South West Ethiopia, factors attributed to high stigma included living in a rural community [17]. This could be due to the high illiteracy rate and lower economic status within rural Ethiopia [17]. Further evidence of stigma in addition to lack of acceptance and support is demonstrated in a case control study in Ethiopia. The study found people suffering from mental health disorders suffer significantly more violence $(28.4 \%)$ in a lifetime versus their counterparts $(17.4 \%)$ within their community or family [18].

Conceptual framework: was opted from World Health Organization mhGAP (fig 1)

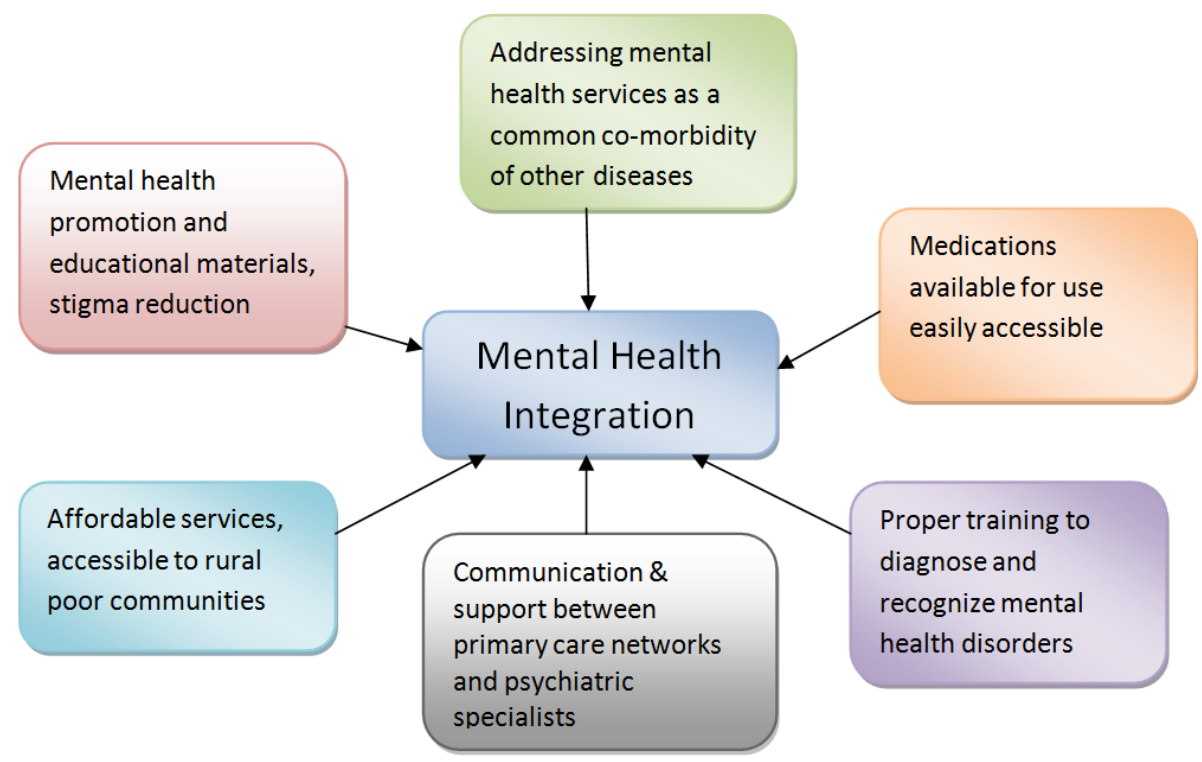

Figure 1. Key factors associated with mental health integration Adapted from concepts of World Health Organization mhGAP 2013 [1].

\subsection{Justification for the Study}

World Health Organization in conjunction with Wonca identified 7 key reasons for addressing mental health disorders as follows [6]: (1) the tremendous burden caused by mental health disorders, (2) the common existence comorbidities for mental and physical health problems, (3) the lack of treatment for millions of people, (4) primary care reaches greater populations, (5) primary care enhances human rights by reducing stigma and discrimination, (6) primary care is affordable, and (7) primary care for mental health leads to good outcomes especially in collaboration with specialists and various levels of care [6].

People seeking help for conditions such as HIV, chronic infections and illnesses may be at higher risk for mental distress and psychiatric disorders. The primary health center may be the easiest way to identify these conditions, as people 
in rural villages often do not seek care. Identifying the current integration level in the primary care system would allow us to determine if more resources are needed to improve mental health.

\subsection{Significance of the Study}

In an effort to reach the goals of the WHO Global Mental Health Action Plan 2013-2020, the Ethiopian Ministry of Health created the Mental Heath Gap Action Plan 2013 (mhGAP). Both plans agree on coordinating services and task-shifting responsibility to allow for a scale up of mental health services throughout the country [1]. According the mhGAP 2013 document, scale up was scheduled to begin in January 2014 and included greater mental health training for primary health care workers [1]. This study would track the progress of the plan and evaluate whether or not mental health services have been incorporated into the primary health care level. The findings will be used by program managers, clinicians in primary health care, policy makers and the community at large.

\section{Objectives}

\subsection{General Objective}

- To determine Level of Mental Health service Integration in Primary Health Care Units based on recommended integration principles in Debre Markos town, northwest Ethiopia, 2014

\subsection{Specific Objectives}

- To determine the level of integration of mental health services in primary care health units

- To compare the implementation of mental health integration in primary health care based on recommended integration principles in the local context

\section{Methods}

\subsection{Study Design}

A cross sectional study design was conducted using a literature review of mental health policy and current integration studies in Ethiopia followed by an interview and document review of a primary health care unit and a health post in the Debre Markos town.

\subsection{Study Area and Period}

In addition, the Debre Markos referral hospital was surveyed for association with the primary health care units and to determine referral utilization. Interviews at each location addressed current policies and procedures regarding mental health services and utilization of services. The Debre Markos population in 2013 was estimated to be approximately 107,684 people [19]. The primary health centers consist of four (4) health centers and ten (10) private clinics. One referral hospital, Debre Markos Hospital, serves the East Gojam Zone. One district health office organizes the 4 health centers and there are no district hospitals within Debre Markos. The data collection was completed on October $22^{\text {nd }}, 2014$ and consisted of information collected from the first six (6) months of 2014.

\subsection{Source Population}

The source population included all primary health care unit heads/managers in Debre Markos town including 4 health centers and 10 private clinics.

\subsection{Study Population}

The study population included primary health care unit heads and managers who fulfilled the inclusion criteria.

\subsection{Eligibility Criteria}

Eligible participants regularly provide primary care services and completed the survey. Recruited participants must be an authorized health official such as a HEW, trained midwife, physician, or facility designee.

\subsection{Exclusion Criteria}

The survey excluded staff members not present the day of collection as well as those unable respond to the questionnaire.

\subsection{Sample Size Determination}

The health care provider sample size included all primary care providers working on the day of the survey.

\subsection{Sampling Technique}

Of the 14 primary health care units in the Debre Markos town, 2 were selected for study. The Debre Markos Hospital was excluded as being a referral hospital and not a primary health care facility. However, the Debre Markos Referral hospital was evaluated for the number of referrals reaching the referral hospital. Health care officers and medical staff working at each health care unit on the day of data collection were included in the study.

\subsection{Variables of the Study}

Dependent variable

- Level of mental health integration (good or poor)

Independent variables

- Mental health conditions as a common co-morbidity to other diseases

- Availability of medications at the primary care unit

- Proper training to diagnose and refer mental health disorders

- Affordable services, access to those services in rural and poor communities

- Mental health promotional materials, stigma reduction

- Level of health institution (health post, health center and hospital)

- Communication and support between primary care 
networks and psychiatric specialists

\subsection{Operational Definition}

Mental Illness: Mental conditions which can affect mood, thinking, and behavior such as schizophrenia, anxiety disorder, depression, and eating disorders [20].

Mental health integration: Linking primary care providers with mental health care providers in order to unify mental health and medical health concerns. Various plans and methods exist to attempt this strategy [21].

\subsection{Data Collection Procedure}

Selected health officials who interacted with patients to provide advice or treatment were asked to participate. Information was collected regarding components of mental health services, frequency, and availability of care. In addition, the officials in charge of patient records were asked if documents could be reviewed. This occurred on the day of data collection.

Data related to program implementation procedures was collected through a survey questionnaire administered to health officials at the designated health facilities within Debre Markos town. Patient records were examined manually; cases of visits for mental health were documented. A record count on qualitative data was translated from the local language (Amharic) and documented on a second survey record sheet. Literature was collected through obtaining government policies on mental health and peer review journals on mental health integration and associated factors in Ethiopia.

\subsection{Data Quality Control}

The data collection tools were reviewed by a Public Health professional of Debre Markos University in order to check for errors and clarity as well as correct translation into Amharic then translated in to English by a local language speaker.

\subsection{Data Processing and Analysis}

Analysis was based on two (2) categorized integration levels: poor integration and good integration. Based on the data collected, the level of integration was determined for each primary health care unit as well as the Debre Markos primary health care centers as a whole. Data from the Debre Markos referral hospital was used to determine if a significant number of referral patients proceed to the next level of care.

\subsection{Ethical Consideration}

Ethical approval will be obtained from the Global Health Coordinator from Touro University and the Public Health Department of Debre Markos University. Surveys completed by health officials will remain anonymous and only include a location. Personal information about health officials, other than job title, was not collected.

\section{Results and Discussion}

\subsection{Data from Debre Markos Health Post \& Health Center (Primary Care)}

Approximately 30 patients were seen in the health center in the last 6 months. Behavioral health and medical care documentation are kept together in the town health center and in the health post. In the health post there were about 9 mental health patients from which 7 of them were epileptic. It is important to consider that some patients may be double counted since there was no organized record system. All records were documented on paper charts.

Referred patients were not correctly registered, but the health extension workers reported they may refer cases to an advanced health facility similarly to other non mental health referrals. The health post and health center did not have an organized method for recording patient data; primary care staffs only record the case and send the patient to the next referral level (health center or referral hospital). A physician was not available in the primary care center or health post; however public health officers serve as physicians in the health center. Mental health promotional materials were not available. Most of the mental health problems were epilepsy and very rare cases of substance or alcohol abuse. Services such as support groups for substance abuse were not available.

While mental health was addressed in the primary care units, there was not a systematic or organized way of keeping records or communicating with mental health specialists. The health extension workers were familiar with HIV/AIDS patients and epileptic patients as well as substance abuse patients. Due to the high stigma associated with these conditions and lack of social support, it is likely many suffering from mental health conditions do not seek help. The primary care system does not have a method to reach out to patients in the community. The capacity for training and education is limited.

\subsection{Data from Debre Markos Hospital (Secondary Care)}

The Debre Markos referral hospital served 446 patients in the last 6 months from which greater than $60 \%$ of them were epilepsy cases. The remaining cases were from depression and bipolar disorders. The exact numbers were not recorded in the hospital, but visits can reach up to 10 patients per month. There was no psychiatrist specialist in the hospital. Behavioral health documentation and other medical records are kept separately. Promotional and informational materials regarding mental health were not available. Psychiatric drugs were readily available in the hospital through prescription. The price of services may vary when there is regimen change. Stable patients will have a follow up appointment every 2 months. There was no guideline for dealing with mental health, but there were treatment protocols and posters regarding various conditions. The hospital does not hold group sessions on mental health issues. The psychiatric nurses teach patients according to their case. 
Based on these findings, the secondary care system was better equipped to support mental health illnesses. However, there was a lack of communication and guidance between the referral hospital and the primary care centers. Thus, the quality of care for monitoring of referrals and adherence to treatment is low. While the facility, nurses, and medications are available at the secondary care system, there is a gap in coverage from the primary to secondary care levels. Most patients were self-referred, indicating need for services and failure at the primary care level. It is possible many patients are unaware of services at the primary care level and go directly to the hospital. The health posts and health care units also serve a smaller geographical area. The patients may feel there is lack of mental health professionals, materials and medications available at the primary care units.

\subsection{Policies and Planning}

In the Debre Markos health center the senior public health officer was interviewed. The health center did not have separate policies and procedures on referrals between primary health care and behavioral care; patients are referred similarly to other medical cases. In both the health post and the primary health care center there is no established procedure for providing feedback to the referrer. Health extension program workers had only some teaching guide for health education purposes. The health center did not have mental health promotion material except for HIV clinic staff. All of the interviewed health professionals identified the need of promotional materials.

The low resources and shortage of staff available at the Debre Markos health center may be the cause for minimal planning. While a policy is instated at the national level, more efforts to train at the local level are needed. The lack of guidance at the health center shows little evidence for standardized training or care between health officers of each primary health unit. Guidance and planning for HIV patients was more established. All other cases of associated mental health conditions received little planning.

As of 2012, the first national policy on mental health was implemented by the Ethiopia Federal Ministry of Health (FMOH) [22]. The policy and plan titled National Mental Health Strategy is dated to address mental health from the years 2012 through 2016 [22]. The stated policy is adapted from the mental health Gap Action Plan (mhGAP) intervention guide for non-specialty workers written by the World Health Organization [1]. In addition, the current Health Sector Development Plan IV (HSDP IV) planned to integrate mental health into $50 \%$ of healthcare settings by 2015 [23]. By this time, primary care will attempt to target psychosis, depression, epilepsy, and substance abuse disorders [23]. The policies address key issues including: support for attitude and behavior change, training, communication, integration, funding, and evaluation. Studies in collaboration with other countries such as the Netherlands and Sweden in order to further develop policies and planning.

\subsection{Support for Attitude and Behavior Change in Addressing Mental Health Issues}

The Debre Markos health center did not hold groups or training lessons on mental cases separately, but they give health education in general for all patients regarding mental health alongside other health aspects. There was no separate session regarding smoking, alcohol and substance use.

Throughout Ethiopia, stigma is a continuing challenge for mental health patients. Current perceptions for the cause of mental illnesses are often attributed to supernatural powers [22]. A study of schizophrenic patient concluded patients did not want to be seen taking medications for mental health disorders, which led to non-adherence to medication [24]. A significant number of patients described 'feeling better' as a reason for treatment attrition [24]. There were no articles found to document an attempt to alleviate the negative attitudes and stigma associated with mental health disorders.

According to the National Mental Health Strategy, human rights essential to all include the "right to shelter, education, employment without stigma or discrimination" [22]. The FMOH mental health strategy describes human rights for all and lists vulnerable populations such as disabled, elderly, women, and prison populations [22]. Specifics on how to provide these rights are not included.

While policies at the national level aim to support acceptance for mental health conditions between various populations, a commitment is not yet evident at the local level. This may be due to lack of training or funding to support promotional activities which would reduce stigma associated with mental illness. With only a few health extension workers at each health post, the capacity for each worker to address mental health alongside other routine health concerns is limited. Primary care workers have limited training and few materials to advocate for mental health. A standardized guideline for addressing mental health disorders at the health posts and health center would bring awareness to the problem and serve as a tool to promote acceptance and standardized care. To change behavior and attitudes in an integrated system community members and community organizations as well as primary health care units would have to collaborate and develop skills to address mental illness with a positive approach. For example, a support group for substance and alcohol abuse could be organized by community members. Primary care units could refer patients to the support group as needed and even provide professional support.

\subsection{Appropriate Training for Primary Care Workers, Feasibility, Limitations}

Health professionals (health extension workers) working in the health post did not have special mental health training except for those working in HIV/AIDS who received 3 days of training. Training for health extension worker to recognize symptoms of mental illness and refer other conditions such as epilepsy and schizophrenia would advantageous to providing speedy treatment. As there are a greater number of health 
posts than health centers, increased training may also target a greater population. In the health center health officers and nurses can assess risk for violence and aggression, refer patients, treat acute violence and aggression, provide some treatment and follow-up as well as case management [22].

Current training to expand psychiatric services in Ethiopia includes a psychiatry residency program in Addis Ababa University. Graduate psychiatrists work in the regional and referral hospitals, but mid-level workers at district level are needed. Jimma University adopted a similar program for non-physician mental health training implemented January 2010 and funded by the Federal Ministry of Education. Additionally, the program has a plan to coordinate with schools and children with community organizations [25]. Health manuals through the carter center are available in conjunction with Toronto University of Canada [26].

Jimma University changed the graduate program in 2008 to include training for mid level health workers. They were to work in district hospitals and provide supervision and support for primary health clinics to focus on child and adolescent mental health [25].

The national mental health strategy defines specific job duties for various areas of health. The primary health posts, for example, will include prevention, promotion, screening, mental health education, medication monitoring, monitor for signs of relapse, community awareness, anti-stigma campaigning. They will know the basic signs and symptoms of mental illness [22]. Current studies on the implementation and status of education and monitoring are not available.

\subsection{Access to Mental Health Professionals or Mental Health Coordinator for Referrals and Support}

The primary care is located 2 to $3 \mathrm{Km}$ from the referral hospital in the town; however, the other 4 primary care units served by the referral hospital are much further away. None of the health facilities (heath post, health center, and the hospital) had a psychiatrist but the hospital has 4 psychiatric nurses. Mental health care providers and primary care officials seldom communicate. This was evidenced from the hospital, but in the health center and heath posts there was no frequent communication. This may be due to a limited number of staff working on mental health services. Almost all of patients at the Debre Markos referral hospital were self referred. While referrals were made between primary care and secondary care facilities, there was not an organized method to track, trend or evaluate referrals. A defined system for referrals and communication between health professionals may help provide support and coordinate services between facilities.

The FMOH National Mental Health Strategy defines the role of the referral hospital as a support system for lower level facilities [22]. The plan states the regional hospital will have a place for acute stabilization and short stay services [22]. The regional hospitals will provide support for primary health care workers as well as advocate for mental health at the district level [22]. There was no evidence found regarding a mental health service coordinator either through literature or the local setting. According to the psychiatric nurse at Debre Markos referral hospital previous attempts to coordinate mental health services were not sustained because of various logistical reasons. Distance between primary care centers, funding for travel, low utilization of technology such as internet may play a role in the lack of communication. Designating a mental health coordinator for support services would enable ideas to be shared between health care units and create a network of support.

\subsection{Availability and Accessibility of Psychotropic Services for Distribution from Primary Care Facilities}

Limited psychotropic medications were available in the health center, but were not available in the health post; health extension workers are not qualified to prescribe medication. The primary care centers may refer patients to the Debre Markos Referral Hospital where medications for mental health disorders are readily available. The following table displays approximate out of pocket costs for select services at the Debre Markos Referral Hospital (Table 1).

People with mental disorders can afford treatment as evidenced from the health post and health center, but a senior psychiatrist nurse from the hospital reported the opposite answer since patients were not functional (productive) and the medications are expensive. The cost of services and referral at from the primary care health post and health center may be minimal, however treatment is limited. In order to receive specialized care at the referral hospital, the cost may deter patients from seeking treatment or following up on previous treatment.

According to the National Mental Health Strategy, drugs will be available in the primary health care units of urban areas only [22]. The primary health care units will refer patients for treatment [22]. An increase in national funding toward expanding services at the primary care level would lead to greater affordability for treatment and, in turn, a more productive workforce. Improvements to track and monitor distribution of drugs and services could ensure the goals of the national policy come to fruition.

Table 1. Cost of psychiatric services at Debre Markos referral hospital 2014.

\begin{tabular}{lll}
\hline & First Time Appointment & $\begin{array}{l}\text { Follow-Up } \\
\text { Services }\end{array}$ \\
\hline Schizophrenia & 30-40 Birr & 30-55 Birr \\
Depression/Anxiety & 40-50 Birr & 60-65 Birr \\
Substance-Use & No service & No service \\
Epilepsy & 24-30 Birr & 24-40 Birr \\
\hline
\end{tabular}

\subsection{Integration through Developments, Agreement and Acceptance}

At the time of evaluation the Debre Markos primary care units did not have an implemented system for mental health development. Developments either through guidelines, institutional policy or agreed procedures were not evident. The primary health care units, with assistance from the referral hospital, could work together to develop a standardized method of care. Agreement from the primary 
care level could come from sending health professional leaders to the national training programs to share information find new ways to expand the capacity for mental health services.

Most training and development for mental health professionals is limited to the capital city, Addis Ababa [26]. In 2011 a meeting was held in Addis Ababa to discuss appropriate training for prison inmates [26]. In addition, a new hospital will open in Addis Ababa with child services [27]. The National Mental Health Strategy identifies the need for performance monitoring, implementation, and evaluation surveys for the population [22]. Mental health care will be decentralized and close to home as possible; it will be essentially free or 'affordable' for all [22]. This strategy acknowledges the need for expanded services. As of 2010, FMOH has a system to track and monitor the prevalence of mental health cases [22]. However, evidence for policy and performance monitoring could not be found. Continual evaluation of the national strategy would help determine if the policy has reached the desired areas of care and identify gaps for improvement.

\subsection{Involvement of Various Stakeholders Including Community Health Workers, Non-Governmental Organizations, and Volunteers}

Currently, involvement of community volunteers or nongovernmental organizations could not be identified through data collection. In the local setting there is no available fund to support mental health promotional or community and group participation. Involvement of health workers includes mental health limited training, education and referral for conditions such as epilepsy and depression.

According to literature, communities fail to recognize severe depression [22]. Community stakeholders may not be aware of the presence of various mental illnesses within the community. Spreading awareness about identifying factors and ways to support mental health disorders within Ethiopia may lead to greater participation by community groups. In addition, the health workers, non-governmental organizations, and volunteers could work together to create a greater support system for those in need.

\subsection{Financial and Human Resources}

There was no available fund for mental health services in all of the health facilities evaluated in the Debre Markos town. In order to expand capacity for treatment, Debre Markos primary care units would need financial support for treatment services and personnel. Finances could be directed towards promotional materials, expansion of medications, formation of support groups, and communicational meetings between facilities. Additional funding could be used to reduce the treatment and associated fees for patient services.

In a study from the Sodo, Ethiopia region, the medication cost is out of pocket by patients, not accessible in primary health care facilities, and limited to antiepileptic and benzodiazepines (anxiolytic) medications [28]. Amanuel hospital 268 beds, 2013 expected hospital beds. There are 57 psychiatric units in Ethiopia, serviced by psychiatric nurses. Specialized mental health specialists at the general hospitals.

Mental health accounts for $12 \%$ of the disease burden in Ethiopia, yet has never exceeded $2 \%$ expenditure on health care [29]. The FMOH National Mental Health Policy describes an increase in funding to expand facilities. Included in this plan is a new hospital with designated beds for psychiatric services and funding to add additional medications to the list of current drugs and to make medications affordable [22]. Finally, the plan describes the possibility of financing mental health services through a new social health insurance initiative [22]. This initiative has yet to be implemented at the national level. Expansion of funding at the national level should attempt to increase capacity and learning for health extension workers and health officers. The funding should extend beyond the scope of major cities in order to have a lasting impact. The main finding of the study is summarized in table 2 .

Table 2. Integration Factors in Primary Health Care Units in Ethiopia and Debre Markos town, 2014.

\begin{tabular}{|c|c|c|}
\hline Integration Factors & Ethiopia (from literature) & Debre Markos PHC Unit \\
\hline Policies and planning & $\begin{array}{l}\text { National Mental Health Strategy (NMHS) } \\
\text { Health Sector Development Plan IV }\end{array}$ & $\begin{array}{l}\text { No policies at Health Post } \\
\text { No procedures at Health Centre } \\
\text { Limited training for HEWs }\end{array}$ \\
\hline $\begin{array}{l}\text { Support for attitude and } \\
\text { behavior change }\end{array}$ & National Mental Health Strategy describes support for change & $\begin{array}{l}\text { Limited support groups for general health, along with } \\
\text { mental health, at health centre } \\
\text { No support system at health post }\end{array}$ \\
\hline Appropriate training & $\begin{array}{l}\text { Training in Addis Ababa University \& Jimma University } \\
\text { NMHS defines specific roles for Health Centres and Health Posts }\end{array}$ & Health centre training limited to HIV/Aids patients \\
\hline $\begin{array}{l}\text { Access to mental health } \\
\text { professionals }\end{array}$ & $\begin{array}{l}\text { Regional hospitals will provide support to primary care hospitals } \\
\text { No evidence of a mental health coordinator }\end{array}$ & $\begin{array}{l}4 \text { psychiatric nurses available at Debre Markos referral } \\
\text { hospital (not in primary care system) }\end{array}$ \\
\hline $\begin{array}{l}\text { Accessible/available } \\
\text { medications }\end{array}$ & Medication available only in urban primary health centres & $\begin{array}{l}\text { Limited medications in Debre Markos health centre } \\
\text { No medications available in health post }\end{array}$ \\
\hline $\begin{array}{l}\text { Integration through } \\
\text { developments, training }\end{array}$ & $\begin{array}{l}\text { Meetings held in Addis Ababa to discuss training for prison inmates, } \\
\text { children, vulnerable populations }\end{array}$ & Limited training for HEWs in health posts \\
\hline $\begin{array}{l}\text { Involvement of } \\
\text { stakeholders }\end{array}$ & $\begin{array}{l}\text { Federal Ministry of Health, Addis Ababa University, Jimma } \\
\text { University, regional hospitals, Primary care units }\end{array}$ & $\begin{array}{l}\text { Primary health centre and health post have limited } \\
\text { involvement in mental health disorders }\end{array}$ \\
\hline $\begin{array}{l}\text { Financial and human } \\
\text { resources }\end{array}$ & $\begin{array}{l}\text { Funding through FMOH was made to expand facilities and increase } \\
\text { the number of medications available }\end{array}$ & $\begin{array}{l}\text { Funding for mental health services not available } \\
\text { Health officer serves health care centre } \\
\text { HEWs serve health post with limited training }\end{array}$ \\
\hline
\end{tabular}




\section{Limitations}

In collecting data from primary care facilities of Debre Markos, only one primary care health center and one health post were selected for evaluation, which were in close proximity to the Debre Markos referral hospital. The standards of care and training for health officials and health extension workers may differ between facilities and may not provide a representative evaluation for Debre Markos as a whole. Those living in rural areas especially may find difficulty in accessing primary care services. Additionally, the stigma associated with mental illness, the utilization of traditional remedies, and the lack funds by patients may contribute to an underrepresentation of the prevalence of mental health conditions in Debre Markos town. Underreporting by staff or failure to document cases is possible as patient files were not well organized No information to assess involvement of various stakeholders in the Debre Markos town.

\section{Conclusions}

Studies about mental health integration into primary care are limited to urban areas in Ethiopia. This research can be utilized as a source for measuring level of integration in small towns such as Debre Markos. It can also serve as a guide for future interventions by primary care facilities, community organizations and the population at large.

Based on the evidence from literature and data collected in the town, mental health integration into primary care is poor. Mental health appears to be integrated into the primary health care system through documentation and administration of mental health services in conjunction with non-mental health conditions. However, the capacity for intervention, diagnosis, and referral is limited in Debre Markos town. Several mental health topics have yet to be included in training for health workers such as support for substance abuse patients.

Evidence from the literature and policies within Ethiopia suggest the Federal Ministry of Health is aware of the need for integration and is making strides to reach greater populations. The National Mental Health Strategy in Ethiopia is in its infancy, and while many recommendations listed may be beneficial to the population, primary health care units currently lack the capacity, support, and training needed to deliver many mental health services. Facilities must work on education, and finding innovative ways to promote, detect and refer patients. In doing so, utilization of this service may be more widely spread.

Integration of mental health services into primary care will lead to greater accessibility by the underserved community. Better treatment services and follow up will create a better environment for mental stability. In addition, patients will be better equipped to seek treatment for other underlying conditions, reducing the daily adjusted life years lost to disability and illness. In fact, a healthy state of mind will contribute to the social and economic development of the country.

\section{Recommendations}

The local teaching schools such as Debre Markos University (DMU) should participate in community awareness program planning. The DMU public health department could apply for grants to increase funding for activities such as creating promotional materials and community outreach programs. Researchers in public health should do a more in depth study of the prevalence of mental health illnesses in Debre Markos town including the attitude and beliefs about the various disorders. The medical college should conduct trainings related to mental health in order for primary care physicians to enable them to identify, diagnose and, if needed, refer patients to a specialist.

Primary health care units should consult and communicate with the Debre Markos referral hospital mental health professionals for monitoring and advice. The primary care units should make greater efforts to document mental health cases and the number of referrals. It is recommended primary health care facilities work on the scale up of health care services and trainings. At this level, electronic records could benefit the traceability and track ability of given services as well as monitor returning patients.

The Debre Markos referral hospital should communicate with primary care units to ensure health officers receive guidance. Frequent connection could occur through telephone meetings in order to minimize travel costs. The hospital should elect a mental health program coordinator to manage services between various levels of care. The referral hospital should manage the communication among other health care units. The hospital should administer and manage programs such as coordinating awareness programs, campaigns, and opening doors for research by identifying the problems and providing topics and/or areas of study.

The government should create guidelines for trainings and issue them at each tier of health care workers in order to standardize care. Training should extend beyond the scope of epilepsy and HIV/AIDS and also address other stigmatized conditions such as depression substance abuse.

Health professionals should create opportunities for updated training on communication, language, and sensitivity towards patients with mental health disorders. Health professionals in both the primary care system and referral hospital should be proactive in sharing ideas to improve care and seeking support.

Efforts to involve a wider range of stakeholders such as community members through support groups or advocacy for stigma reduction could play a key role in service utilization and outreach to underserved populations. Accountability by all parties should be improved through better organization of patient files as well as periodic evaluation. 


\section{References}

[1] WHO, Mental Health Gap Action Programme (mhGAP): Scaling up care for mental, neurological and substance use disorders. . 2013.

[2] Bitew, T., Prevalence and risk factors of depression in Ethiopia: A review. Ethiopia Journal of Health Sciences, 2014. 24(2).

[3] Worku, D., Epilepsy in Ethiopia. Neurology. 80(3): p. 127.

[4] WHO, Mental health and substance abuse. 2014, World Health Organization.

[5] WHO, WHO-AIMS Report on mental health system in Ethiopia. 2006: Addis Ababa.

[6] WONCA, W.a. Integrating mental health into primary care, A global perspective. 2008.

[7] Kibour, Y.P., Mind the Gap: Personal Reflections on the Mental Health Infrastructure of Ethiopia. American Psychological Association, 2010.

[8] WHO, Comprehensive mental health action plan 2013-2020. 2013.

[9] WHO, Mental Health Atlas 2011. 2011: Italy.

[10] Ervin, D.A.W., Ashley; Merrick, Joav, Primary care: mental and behavioral health and persons with intellectual and developmental disabilities. Frontiers in Public Health, 2014. 2(76).

[11] Shannon, P.J., Refugees' advice to physicians: how to ask about mental health. Family Practice, 2014. 31(4): p. 462-466.

[12] Mousley, E.e.a., The impact of podoconiosis and quality of life. Health and Quality of Life Outcomes, 2013. 11(1): p. 122.

[13] Bifftu, B.B.e.a., Stigma resistance among people with schizophrenia at Amanuel Mental Specialized Hospital Addis Ababa, Ethiopia: a cross-sectional institution based study. BMC Psychiatry, 2014. 14(259).

[14] Girma, E.e.a., Self-stigma among caregivers of people with mental illness: toward caregivers' empowerment. Journal of Multidisciplinary Healthcare, 2014. 2014(7): p. 37-43.

[15] Thornicroft, G. Shunned: discrimination against people with mental illness. 2006 [cited 2014 10/03].

[16] Deribe, K.e.a., Defaulters from antiretroviral treatment in Jimma University Specialized Hospital, Southwest Ethiopia. Tropical Medicine and International Health, 2008. 13(3).

[17] Girma, E.e.a., Public Stigma against People with Mental Illness in the Gilgel Gibe Field Research Center (GGFRC) in Southwest Ethiopia. PLoS ONE, 2013. 8(12).

[18] Tsigebrhan, R.e.a., Violence and violent victimization in people with severe mental illness in a rural low-income country setting: A comparative cross-sectional community study. 2013.

[19] University, D.M. Short History of Debre Markos Town. 2014; Available from: http://web.dmu.edu.et/test-dmusite/index.php/category-comments/debre-markos.

[20] Clinic, M. Diseases and Conditions: Mental Illness Definition. 2014 [cited 2014 10/05]; Available from: http://www.mayoclinic.org/diseases-conditions/mentalillness/basics/definition/con-20033813

[21] Butler, M.e.a., Integrating Mental Health/Substance-Abuse and Primary Care. 2008.

[22] FMOH, National Mental Health Strategy, E. Federal Ministry of Health, Editor. 2012.

[23] Fekadu , A., Graham Thornicroft, Global Mental Health: Perspectives from Ethiopia. Global Health Action, 2014. 7(25447).

[24] Teferra, S.e.a., Perspectives on reasons for non-adherence to medication in persons with schizophrenia in Ethiopia: a qualitative study of patients, caregivers and health workers. BMC Psychiatry, 2013. 13(168).

[25] Tesfaye, M.e.a., The development of a model of training in child psychiatry for non-physician clinicians in Ethiopia. Child \& Adolescence Psychiatry \& Mental Health, Biomed Central 2014. 8(6).

[26] Center, T.C. Mental Health. 2014 11/1/2014]; Available from: http://www.cartercenter.org/health/mental_health/index.html.

[27] Fricchione, G.L.e.a., Capacity Building in Global Mental Health: Professional Training. Harv Ref Psychiatry, 2011. 2012(20): p. 47-57.

[28] Hanlon, C.e.a., Challenges and Opportunities for Implementing Integrated Mental Health Care: A District Level Situation Analysis from Five Low- and Middle-Income Countries. PLoS ONE, 2014. 9(2).

[29] Chemali , Z.N., Christina PC Borba, Tanya E. Henderson, Markos Tesfay, Making strides in women's mental health care delivery in rural Ethiopia: demographics of a female outpatient psychiatric cohort at Jimma University Specialized Hospital (2006-2008). International Journal of Womens Health, 2013. 2013(5): p. 413-419. 